\title{
ESTUDIOS DE RADIODIAGNÓSTICO DURANTE EL EMBARAZO
}

\author{
Reinaldo Uribe SM. ${ }^{1}$, Nicolás Sáez O. ${ }^{2}$, Jorge Carvajal C. ${ }^{3}$, PhD. \\ ${ }^{1}$ Médico General, Pontificia Universidad Católica de Chile. ${ }^{2}$ Programa de Obstetricia y Ginecología, ${ }^{3}$ Departamento de \\ Obstetricia y Ginecología, Escuela de Medicina, Pontificia Universidad Católica de Chile.
}

\section{RESUMEN}

A pesar de la creciente utilización de estudios radiológicos durante el embarazo, continúan existiendo percepciones erróneas respecto a posibles riesgos materno/fetales de su uso. En esta revisión presentamos la evidencia relevante sobre los efectos potenciales y la seguridad clínica de la exposición a radiación en estudios diagnósticos durante el embarazo. La mayoría de los exámenes exponen al feto a radiación en dosis menores a $50 \mathrm{mSv}$, las que han demostrado no ser dañinas. Los medios de contraste yodados pueden producir efectos deletéreos en la glándula tiroides del feto en desarrollo. Para el gadolinio existe limitada experiencia, por lo que no se recomienda su uso, a menos que el beneficio justifique los riesgos potenciales. Tanto la ultrasonografía como la resonancia magnética son seguros para el feto, por lo que debieran ser preferidos. Recomendamos descartar embarazo antes de efectuar un estudio de radiodiagnóstico a una mujer en edad fértil, con el objetivo de no exponer a los fetos a radiación ionizante de manera no justificada. Sin embargo, cuando la necesidad diagnóstica y el tratamiento asociado lo requieran, el examen debe ser solicitado de manera usual informando a los pacientes los potenciales riesgos y beneficios.

\section{PALABRAS CLAVE: Radiodiagnóstico, radiación ionizante, embarazo}

\section{SUMMARY}

Despite the increasing use of radiologic test during pregnancy, there are still wrong perceptions about maternal and fetal risks. Here we present relevant evidence about safety and potential side effects of radiation exposure in diagnostic imaging during pregnancy. Most $\mathrm{x}$-ray studies expose the fetus to doses lower than $50 \mathrm{mSv}$, that have proven to be harmless. lodine-based contrast media may damage the developing fetuses' thyroid gland. Experience in gadolinium use is limited, thus its use is not recommended, unless the potential benefit worth its potential risk. Both ultrasound and magnetic resonance are safe to the fetus and they should be preferred over other techniques. We recommend discarding pregnancy before proceeding with any radiodiagnostic study in women of reproductive age, in order to prevent unnecessary fetal exposure to ionizing radiation. Nevertheless, when clinically justified, the x-ray study must be performed as usual, informing the patients about the potential risks and benefits.

KEY WORDS: Radiodiagnosis, ionizing radiation, pregnancy 


\section{INTRODUCCIÓN}

Con frecuencia en la práctica clínica obstétrica es necesario exponer a radiación ionizante a mujeres embarazadas, como parte de estudios de radiológicos indispensables para el diagnóstico de patología médica asociada. Surge en estos casos, la preocupación por la cantidad de radiación fotónica que recibe el embrión o feto y las consecuencias que esta exposición puede ocasionar en su desarrollo.

Aquí presentamos la evidencia relevante, acumulada principalmente por el Colegio Americano de Radiología (American College of Radiology: ACR), la Comisión Internacional de Protección Radiológica (International Commission on Radiological Protection: ICRP) y el Colegio Americano de Obstetras y Ginecólogos (American College of Obstetricians and Gynecologists: ACOG), sobre los efectos potenciales y la seguridad clínica de la exposición a radiación, en estudios diagnósticos durante el embarazo, para facilitar la labor de obstetras y médicos generales o especialistas, en la decisión de solicitar el examen apropiado al paciente que se atienda.

\section{CONCEPTOS SOBRE RADIACIÓN IONIZANTE}

La radiación ionizante puede ocasionar la muerte celular y el daño permanente sobre el ADN como fenómeno inicial en la carcinogénesis. Ambos efectos están directamente relacionados a la dosis de radiación a la que se expone un ser vivo (1).

La dosis de radiación absorbida por el embrión o feto se expresa en unidades de medida que han cambiando a través de los años (2). El Rad (rad) es la abreviatura de las palabras inglesas Roentgen Absorbed Dose, que corresponde a la unidad del sistema inglés que mide la dosis de radiación ionizante absorbida por un material. Equivale a la energía de 100 ergios por gramo de materia irradiada. En Estados Unidos, todavía se utiliza el rad, pero en el resto del mundo ha sido sustituido por la unidad del Sistema Internacional (SI) llamada Gray (Gy), que corresponde a 1 Joule (J) de energía absorbida por kilogramo de materia, 1 Gy equivale a $100 \mathrm{rad}$.

Por otra parte, los Roentgen Equivalent Man (Rem) corresponden a la unidad física utilizada antiguamente, y en la actualidad por los países anglosajones, para indicar la peligrosidad de una radiación. La unidad admitida en el SI para medir esta cantidad es el Sievert (Sv) con las mismas dimensiones que el Rem. El Sv es la unidad utilizada para medir diferentes magnitudes en temas de protección radiológica. Mide la dosis de radiación absorbida por la materia viva, corregida por los posibles efectos biológicos producidos.

Se cumple la equivalencia $1 \mathrm{~Sv}=1$ Gy para las radiaciones electromagnéticas (rayos $X$ y gamma) y los electrones, pero es muy diferente para otras radiaciones (20 veces mayor para la radiación alfa, de 1 a 20 para neutrones) (Tabla I).

Tabla I

UNIDADES DE MEDIDA DE RADIACIÓN IONIZANTE

\begin{tabular}{|c|c|c|c|}
\hline Medida & Definición & $\begin{array}{l}\text { Unidad } \\
\text { Inglesa }\end{array}$ & Unidad SI \\
\hline Exposición & $\begin{array}{l}\text { Número de iones } \\
\text { producidos por los } \\
\text { rayos } \mathrm{X} \text { y } \\
\text { absorbidos por } \mathrm{Kg} \\
\text { de aire. }\end{array}$ & Roentgen (R) & Roentgen (R) \\
\hline Dosis & $\begin{array}{l}\text { Cantidad de } \\
\text { radiación absorbida } \\
\text { por Kg de materia. }\end{array}$ & $\operatorname{Rad}(\mathrm{rad})$ * & $\begin{array}{l}\text { Gray (Gy) * } \\
1 \mathrm{~Gy}=100 \mathrm{rad}\end{array}$ \\
\hline $\begin{array}{l}\text { Dosis efectiva } \\
\text { relativa }\end{array}$ & $\begin{array}{l}\text { Cantidad de } \\
\text { radiación absorbida } \\
\text { por Kg de materia } \\
\text { viva corregida por } \\
\text { efecto biológico. }\end{array}$ & $\begin{array}{l}\text { Roentgen } \\
\text { equivalents } \\
\text { man (rem) * }\end{array}$ & $\begin{array}{l}\text { Sievert }(\mathrm{Sv}) \\
1 \mathrm{~Sv}=100 \mathrm{rem}\end{array}$ \\
\hline
\end{tabular}

\footnotetext{
* Para diagnóstico por rayos $\mathrm{X}, 1 \mathrm{rad}=1 \mathrm{rem}$ ó $1 \mathrm{~Gy}=1 \mathrm{~Sv}$.
} 
UMBRAL PARA DOSIS DE RADIACIÓN DURANTE EL EMBARAZO

En término generales se ha establecido que no existe evidencia de riesgo aumentado de anomalías fetales, retardo mental, restricción de crecimiento, o pérdida del embarazo a consecuencia de dosis de radiación ionizante menor que $50 \mathrm{mGy}$ (5 rads).

El riesgo de daño al feto depende de 3 factores principales. La dosis absorbida, la distribución de ésta en el tiempo y la edad gestacional. Por este motivo el análisis se realiza dependiendo de cada etapa del embarazo (3):

-Etapa preimplantatoria (primeras 2 semanas de desarrollo embrionario): en animales experimentales se ha estimado que dosis de 100 a $200 \mathrm{mSv}$ (10 a 20 rem) inducen entre 1 a $2 \%$ de casos de muerte embrionaria. Algo difícil de conceptualizar teniendo en cuenta la alta tasa de abortos espontáneos en este período del desarrollo.

- Etapa de organogénesis (3 a 8 semanas): estudios en animales demuestran que la dosis mínima para causar malformaciones corresponde a 500 $\mathrm{mSv}$ (50 rem), por lo que en humanos se ha mantenido un umbral conservador de 100 a 200 mSv (10 a 20 rem).

- Etapa fetal temprana (9 a 25 semanas): los estudios realizados en los sobrevivientes de Hiroshima, demuestran que el efecto más importante detectado es el retraso mental. La dosis umbral, por debajo de la cual no se manifiesta este efecto esta com- prendida entre los 120 a $200 \mathrm{mSv}$ (12 a 20 rem). - Etapa fetal tardía: en el último trimestre no se espera una mayor incidencia de malformaciones o retraso mental a causa de la radiación. Sin embargo, existiría la probabilidad de desarrollar cáncer en los próximos años. Este hecho ha sido estudiado encontrándose que a dosis menores de 100 mGy (10 rads) la probabilidad de desarrollar cáncer o malformaciones es baja (4) (Tabla II).

Por este motivo, la ICRP sugiere que para dosis fetales menores a 100 mGy, no existe justificación médica para interrumpir el embarazo debido a la exposición a radiaciones. Mientras que el ACR, sugiere considerar intervenir el embarazo, en presencia de otros riesgos, cuando la irradiación al feto se produjo en las primeras 8 semanas y la dosis supera los 50 mGy. Ambas cifras están muy por encima de las dosis de radiación recibidas mediante estudios de radiodiagnóstico $(3,5,6)$.

\section{ESTUDIOS DE RADIODIAGNÓSTICO}

La ansiedad originada por estudios radiodiagnósticos en las embarazadas es debida principalmente, a la falta de información sobre los posibles riesgos para el feto en desarrollo. Es labor del médico dar a conocer los bajos riesgos que implican estos procedimientos, informar la magnitud de las radiaciones que afectarán al embrión o feto y otorgar un ambiente de calma y confianza a la paciente.

Tabla II

PROBABILIDAD DE OBTENER NIÑOS SANOS EN FUNCIÓN DE LA DOSIS DE RADIACIÓN RECIBIDA DURANTE EL EMBARAZO

\begin{tabular}{ccc}
\hline $\begin{array}{c}\text { Dosis absorbida por el } \\
\text { embrión o feto en mGy } \\
\text { (equivalente en rads) }\end{array}$ & $\begin{array}{c}\text { Probabilidad de que } \\
\text { el niño NO tenga } \\
\text { malformaciones (\%) }\end{array}$ & $\begin{array}{c}\text { Probabilidad de que el niño } \\
\text { NO desarrolle cáncer entre los } \\
\text { O-19 años (\%)* }\end{array}$ \\
\hline $0(0)$ & 97 & 99,7 \\
$0,5(0,05)$ & 97 & 99,7 \\
$1(0,1)$ & 97 & 99,7 \\
$2,5(0,25)$ & 97 & 99,7 \\
$5(0,5)$ & 97 & 99,7 \\
$10(1)$ & 97 & 99,6 \\
$50(5)$ & 97 & 99,4 \\
$100(10)$ & 97 & 99,1 \\
\hline
\end{tabular}

\footnotetext{
*Valores aproximados. Se asume de manera conservadora que el riesgo de cáncer fatal, debido a la radiación, es del 0,6\% por 100 mGy de dosis fetal, lo que corresponde a 1/17.000 por mGy, y una relación dosis-respuesta lineal. Muchos estudios epidemiológicos sugieren que el riesgo puede ser aún menor que el asumido aquí. El riesgo natural de cáncer infantil fue calculado a partir de NCI-SEER (1994).
} 
Destaca que la mayoría de las aplicaciones médicas que exigen tomar decisiones y que emplean rayos $\mathrm{X}$ irradian bajas dosis, recomendándose que la estimación de la dosis fetal no es necesaria, salvo cuando el feto se encuentra en el haz directo de radiación (6). Un estudio realizado en el Reino Unido da cuenta de las dosis para los distintos procedimientos, mostrando especialmente como han disminuido significativamente sus dosis desde el año 1993 a 1998 gracias a los avances tecnológi$\cos (7)$ (Tabla III).

Una buena técnica al irradiar al paciente es fundamental. El factor técnico de mayor impacto en la dosis de radiación en las tomografías computarizadas (TC) es la corriente de tubo. Justamente éste es el parámetro de máxima arbitrariedad seleccionado por el operador $(8,9)$, con un rango de $150 \mathrm{a}$ $240 \mathrm{~mA}$ para cortes de 5 a $15 \mathrm{~mm}$ de rotación que representan un rango de dosis al paciente de 7 a 21 mGy por corte helicoidal de $360^{\circ}(10)$.

Existe una gran variedad de medios de contraste $\mathrm{y}$ son utilizados junto con los rayos $\mathrm{X}$ y resonancias magnéticas. Estudios en animales han demostrado que al parecer los derivados del yodo no tendrían efectos teratogénicos (11). Sin embargo, en humanos se han asociado al desarrollo de hipotiroidismo neonatal (12). Además, los estudios de radiodiagnóstico que utilizan medios de contraste tienen una mayor exposición a radiación por necesitarse visiones pre y pos administración del medio. Estos agentes pasan a la leche materna pero no se han asociado a problemas durante la lactancia (11). Generalmente los medios de contraste son utilizados en el embarazo sólo si se justifican sus beneficios y están por sobre sus potenciales riesgos.

En la medicina nuclear los efectos sobre el embrión o el feto dependen de las propiedades físicas y bioquímicas de los distintos radioisótopos. El Tecnecio $99 \mathrm{~m}$ es uno de los más utilizados y resulta en una exposición menor a 5 mGy $(13,14)$. Por ejemplo en el estudio de ventilación y perfusión pulmonar la radiación a la que se expone al feto es extremadamente baja con 0,5 mGy (15). Distinto es con los radioisótopos utilizados para el tratamiento

\section{Tabla III}

\section{DOSIS FETAL ESTIMADA MEDIA Y MÁXIMA, SEGÚN EL TIPO DE ESTUDIO Y EN FUNCIÓN DEL} AÑO EN QUE SE REALIZÓ EL ESTUDIO

\begin{tabular}{|c|c|c|c|c|}
\hline \multirow[t]{2}{*}{ Examen } & \multicolumn{4}{|c|}{ Dosis Fetal en mSv (equivalencia en rem) } \\
\hline & Media & Máxima & Media & Máxima \\
\hline $\begin{array}{l}\text { Radiología } \\
\text { convencional }\end{array}$ & \multicolumn{2}{|c|}{1993} & \multicolumn{2}{|c|}{1998} \\
\hline Abdomen (sólo AP) & $2,6(0,26)$ & $18(1,8)$ & $1,4(0,14)$ & $4,2(0,42)$ \\
\hline Enema de Bario & $16(1,6)$ & $80(8)$ & $5,8(0,58)$ & $24(2,4)$ \\
\hline Papilla de Bario & $2,8(0,28)$ & & $1,1(0,11)$ & $5,8(0,58)$ \\
\hline Tórax & $<0,01(\sim 0)$ & $<0,01(\sim 0)$ & $<0,01(\sim 0)$ & $<0,01(\sim 0)$ \\
\hline Urografía intravenosa & $3,2(0,32)$ & $20(2)$ & $1,7(0,17)$ & $10(1)$ \\
\hline Columna lumbar & $3,2(0,32)$ & $12(1,2)$ & $1,7(0,17)$ & $10(1)$ \\
\hline Pelvis & $1,7(0,17)$ & $8(0,8)$ & $1,1(0,11)$ & $4(0,4)$ \\
\hline Cráneo & $<0,01(\sim 0)$ & $<0,01(\sim 0)$ & $<0,01(\sim 0)$ & $<0,01(\sim 0)$ \\
\hline Columna dorsal & $<0,01(\sim 0)$ & $<0,01(\sim 0)$ & $<0,01(\sim 0)$ & $<0,01(\sim 0)$ \\
\hline Tomografía computada & \multicolumn{2}{|c|}{1993} & \multicolumn{2}{|c|}{1998} \\
\hline Abdomen & $8(0,8)$ & $49(4,9)$ & $8(0,8)$ & $49(4,9)$ \\
\hline Tórax & $0,06(0,006)$ & $0,95(0,095)$ & $0,06(0,006)$ & $0,95(0,095)$ \\
\hline Cabeza & $<0,005(\sim 0)$ & $<0,005(\sim 0)$ & $<0,005(\sim 0)$ & $<0,005(\sim 0)$ \\
\hline Columna lumbar & $2,4(0,24)$ & $8,5(0,85)$ & $2,4(0,24)$ & $8,5(0,85)$ \\
\hline Pelv is & $25(2,5)$ & $79(7,9)$ & $25(2,5)$ & $79(7,9)$ \\
\hline Pelvimetría & $0,2(0,02)$ & $0,4(0,04)$ & $0,2(0,02)$ & $0,4(0,04)$ \\
\hline
\end{tabular}


del hipertiroidismo los cuales están contraindicados durante el embarazo. No obstante, para el estudio diagnóstico de problemas de la tiroides se reemplaza el I131 por I123 o Tc 99m (13).

\section{CONCLUSIONES}

La mayoría de los exámenes diagnósticos que exponen al feto a radiación ionizante le aportan dosis menores a $50 \mathrm{mSv}$ (5 rems), las que no han demostrado ser dañinas. Dado que la evidencia sugiere que sobre 100 mGy (10 rads) existe riesgo de malformaciones, no se debiera exponer al feto a niveles elevados de radiación. Podría existir un pequeño aumento en el riesgo de cáncer durante la infancia de 1 en 2000 en niños expuestos a radiación ionizante, contra 1 en 3000 en la población infantil general.

Tanto la ultrasonografía como los estudios de resonancia magnética $(16,17)$ con un campo magnético de 1,5 Tesla o menos son seguros para el feto, por lo que debieran ser preferidos ante los estudios que lo exponen a elevadas dosis de radiación ionizante.

Los medios de contraste yodados cruzan la placenta y podrían producir efectos en la glándula tiroides en desarrollo del feto, aunque no se han reportado secuelas clínicas a exposiciones breves. El gadolinio es el medio de contraste más usado en resonancia magnética y también atraviesa la placenta. Dada la limitada experiencia con este agente, no se recomienda su uso a menos que el beneficio justifique los riesgos potenciales al feto (18).

Sugerimos seguir las siguientes recomendaciones:

- Antes de efectuar un estudio de radiodiagnóstico a una mujer en edad fértil, siempre preguntar por el riesgo de posible embarazo y de existir éste, realizar un test de embarazo rápido en orina.

- No exponer a los fetos a radiación ionizante de manera no justificada. Sin embargo, cuando la necesidad diagnóstica y el tratamiento asociado lo requieran, el examen radiológico debe ser solicitado de modo usual.

- Informar a toda madre sometida a un examen que la expone a radiación ionizante que los niveles utilizados en la actualidad exponen al feto a un riesgo teórico realmente bajo de muerte, malformaciones, y/o desarrollo de cáncer en los años siguientes.

- Explicar a la madre que en todo embarazo existe la probabilidad, calculada en base a poblaciones, de aborto, malformaciones congénitas, enfermedades genéticas y restricción del crecimiento (aproximadamente $13 \%$; $4 \%$; $10 \%$; y $10 \%$, respectivamente). - Siempre preferir el examen que, aportando buena sensibilidad diagnóstica, exponga al feto a la menor cantidad posible de radiación ionizante.

- Utilizar protección abdominal para minimizar la dosis de radiación ionizante al feto.

- Preferir centros de radiodiagnóstico confiables para evitar la repetición de exámenes, que además del costo, obligarían exponer al feto al doble de radiación ionizante.

- Evitar el uso de medios de contraste que atraviesan la placenta como el gadolinio y los medios de contraste yodados.

\section{BIBLIOGRAFÍA}

1. Brent RL. The effect of embryonic and fetal exposure to x-ray, microwaves, and ultrasound: counseling the pregnant and nonpregnant patient about these risks. Semin Oncol 1989;16:347-68.

2. Rickards J, Cameras R. Unidades que se usan en la seguridad radiológica. En: Las Radiaciones II. Manejo de las Radiaciones Nucleares. 1a edición. México. Fondo de Cultura Económica, 2002;28-30.

3. International Commission on Radiological Protection (ICRP). $N^{\circ} 84$ Ed. SEPR y SAR. Pregnancy and Medical Radiation (2002).

Hallado en: http://www.icrp.org/educational_area.asp. Acceso el 12 de septiembre de 2008.

4. NCl-SEER (1994) Surveillance, Epidemiology and End Results Cancer Statistics Review 1973-1991. National Cancer Institute, Bethesda MD, USA.

Hallado en: http://www.sar.radioproteccion.org.ar/ICRPder.htm. Acceso el 12 de septiembre de 2008.

5. Gray JE. Safety (risk) of diagnostic radiology exposures. En: Janower ML, Linton OW (eds). Radiation risk: a primer. Reston, VA: American College of Radiology, 1996;15-7.

6. Guidelines for diagnostic imaging during pregnancy. ACOG Committee Opinion $n^{\circ} 299$. American College of Obstetricians and Gynecologists. Obstet Gynecol 2004;104:647-51.

7. Sharp C, Shrimpton JA, Burry RF. Diagnostic medical exposures: advice on exposure to ionizing radiation during pregnancy. National Radiological Protection Board, Chilton, Didcot, Oxon, UK. (1998)

Hallado en: http://www.e-radiography.net/regsetc/infozone.htm. Acceso el 12 de septiembre de 2008.

8. Vergara M, Castro M, Matas J, et al. Reducción de dosis al paciente en TC. Un estudio de resultados en la práctica clínica. Rev Chil Radiol 2003;9:29-32.

9. Vergara M. Dosis al feto en radiodiagnóstico. Rev Chil Radiol 2005;11:30-1.

10. Sheck RJ, Coppenrath EM, Kellner MW, et al. Radiation dose and image quality in spiral computed tomography: multicentre evaluation at six institutions. $\mathrm{Br} \mathrm{J}$ Radiol 1998;71:734-44.

11. Morisetti A, Tirone P, Luzzani F, de Haen C. Toxicological safety assessment of iomeprol, a new X-ray contrast agent. Eur J Radiol 1994;18:S21-31.

12. Mehta PS, Metha SJ, Vorherr H. Congenital iodide goiter and hypothyroidism: a review. Obstet Gynecol 
Surv 1983;38:237-47.

13. Cunningham FG, Gant NF, Leveno KJ, et al. General considerations and maternal evaluation En: Williams Obstetrics. 21st ed. New York (NY): McGraw-Hill, 2001;1143-58.

14. Mettler FA Jr, Guiberteau MJ. Essentials of nuclear medicine imaging. 4th ed. Philadelphia (PA): WB Saunders, 1998.

15. Ginsberg JS, Hirsh J, Rainbow AJ, Coates G. Risks to the fetus of radiologic procedures used in the diagnosis of maternal venous thromboembolic disease. Thromb Haemost 1989;61:189-96.
16. Newnham JP, Doherty DA, Kendall GE, et al. Effects of repeated prenatal ultrasound examinations on childhood outcome up to 8 years of age: follow-up of a randomised controlled trial. Lancet 2004;364:203844.

17. Kirkinen $P$, Partanen $K$, Vainio $P$, Ryynanen M. MRI in obstetrics: a supplementary method for ultrasonography. Ann Med 1996;28:131-6.

18. Chen M, Coakley F, Kaimal A, Laros R. Guidelines for Computed Tomography and Magnetic Resonance Imaging Use During Pregnancy and Lactation. Obstet Gynecol 2008;112:333-40. 